\title{
Inability of NCoR/SMRT to repress androgen receptor transcriptional activity in prostate cancer cell lines
}

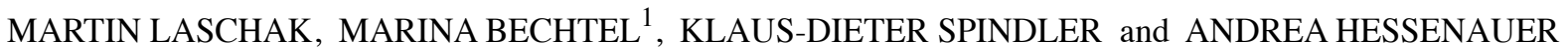 \\ Institute of General Zoology and Endocrinology, Ulm University, D-89069 Ulm, Germany
}

Received April 6, 2011; Accepted May 19, 2011

DOI: $10.3892 /$ ijmm.2011.735

\begin{abstract}
The molecular mechanisms leading to castrationresistant prostate cancer (CRPC) are poorly understood. Among several mechanisms leading to CRPC growth a dysregulation of androgen receptor (AR) co-regulators (i.e. up-regulation of co-activators or down-regulation of co-repressors) is discussed. There are numerous reports demonstrating an increased expression of co-activators during prostate cancer progression. On the contrary, the impact of co-repressors on tumor growth and development is less clear. In this study we compared the effects of two known co-repressors, NCoR and SMRT, on AR transcriptional activity in prostate cancer $(\mathrm{PCa})$ cell lines and compared them to that in COS-1 cells. Interestingly, we found that NCoR/SMRT overexpression did not repress AR-dependent gene expression in the PCa cell lines, but rather activated it. This finding is probably due to an impaired AR-co-repressor interaction in the prostate cancer cell lines. In conclusion, we provide evidence that up-regulation of NCoR or SMRT may increase transcriptional activity of the AR in a cell type-specific context.
\end{abstract}

\section{Introduction}

Prostate cancer ( $\mathrm{PCa}$ ) is the most commonly diagnosed cancer and the second leading cause of cancer-related deaths in elderly men of the western industrialized countries (1). Initially, PCa cells are largely dependent on androgens for growth and survival. Unfortunately, androgen ablation therapy causes only a temporary reduction in tumor cell growth and castrationresistant prostate cancer (CRPC) cells almost invariably develop within a period of around 2 years. The mechanisms leading to

Correspondence to: Martin Laschak, Institute of General Zoology and Endocrinology, Ulm University, Albert-Einstein-Allee 11, D-89069 Ulm, Germany

E-mail: martin.laschak@uni-ulm.de

Present address: ${ }^{1}$ Department of Radiation Oncology, Division of Radiobiology and Molecular Environmental Research, University of Tuebingen, Röntgenweg 11, D-72076 Tübingen, Germany

Key words: co-repressor, prostate cancer, androgen receptor, NCoR, SMRT
CRPC cells are poorly understood. As shown by several clinical studies the androgen receptor (AR) is rarely lost in CPRC cells in vivo (2). Among several mechanisms allowing CRPC to grow under castrate levels of circulating androgens one possibility is the dysregulation of AR cofactors (3). Cofactors are proteins that interact with transcription factors thereby up- (co-activators) or down-regulating (co-repressors) their transcriptional activity. Two members of the p160 co-activator family, namely SRC-1 (steroid receptor co-activator 1) and TIF-2 (transcriptional intermediary factor 2), were shown to be overexpressed in recurrent prostate cancer as compared to benign prostatic hyperplasia $(\mathrm{BPH})$ or androgen-dependent PCa $(4,5)$. In addition to the up-regulation of co-activators, down-regulation of co-repressors has also been suggested to contribute to tumor progression (6). Unfortunately the role of AR co-repressors during prostate cancer progression is unclear (7). Recently Hey1, a transcriptional repressor involved in Notch signaling, was found to also be an AR co-repressor. In BPH tissue, Heyl co-localized with AR in the cytoplasm as well as in the nucleus of epithelial cells. In prostate cancer tissue, Heyl localization was predominantly restricted to the cytoplasm. This localization is thought to abolish the repressive function of Heyl on AR transcriptional activity (8). Prohibitin, another repressor of AR signaling, was shown to be downregulated by androgen treatment. This observation indicates that the AR is able to increase its own activity in tumors by regulating co-repressor expression $(9,10)$. Contrarily, increased prohibitin mRNA and protein levels were detected in prostate cancer compared to BPH (11). Moreover, the well known co-repressor SMRT (silencing mediator of retinoic acid and thyroid hormone receptor) was found to be elevated in prostate cancer cell lines. SMRT mRNA expression was reported to be increased in PC-3 and DU-145 in comparison to normal prostate epithelial cells as well as to the majority of primary PCa cell cultures (12). A higher expression of both SMRT and NCoR (nuclear receptor co-repressor) was found in a CWR22Rv1 sub-cell line with a decreased vitamin D sensitivity (13).

Both, NCoR and SMRT, recruit histone deacetylases and form large multiprotein complexes (14). Different isoforms of the co-repressors have been described. For example, RIP13a is generated by alternative splicing of the NCoR mRNA. SMRT $\alpha$, SMRT $\beta$ and SMRT 2 are splicing products of SMRT mRNA (15). The co-repressors, NCoR and SMRT were shown to interact with the AR, thereby repressing AR transcriptional activity (16-18). 
Despite the fact that NCoR was shown to differentially repress AR activity in COS and HeLa cells (17) the vast majority of experimental studies have been performed in COS and CV-1 cells. In consequence we compared the effects of NCoR and SMRT on AR transcriptional activity in COS- 1 as well as in different PCa cell lines. Interestingly, we found that $\mathrm{NCoR}$ as well as the related co-repressor SMRT are not able to repress AR-dependent reporter gene expression in prostate cancer cell lines. We could also demonstrate an impaired interaction of the NCoR N-terminus with the AR. Thus, we propose that the inability to repress AR transcriptional activity is probably due to this different AR-NCoR binding in PCa cells.

\section{Materials and methods}

Plasmids. The expression plasmid pSG5-AR coding for a wildtype AR was kindly provided by Professor Culig (Innsbruck Medical University, Innsbruck, Austria). Expression constructs coding for green fluorescent SMRT - and NCoR-fusion proteins (pCMV-GFP-SMRT $\tau$ and pCMV-GFP-NCoR (19) were a generous gift from Professor Privalsky (University of California Davis, CA, USA). pCMX-NCoR (20) encoding a Flag-tagged NCoR-Gal4 fusion protein was obtained from Professor Heinzel (Friedrich Schiller University of Jena, Germany). SMRT $\alpha$ and SMRT $\beta$ expression constructs (pCMX-mSMRTaFL and pCMX-mSMRTbFL) were kindly provided by Professor Evans (Salk Institute for Biological Studies, La Jolla, CA, USA). Luciferase reporter plasmids PSA61-luc, pGL3E-Probasin and ARE-luc were provided by Professor Culig (Innsbruck Medical University) and Professor Weidemann (Ulm University, Germany) (21). The pGL4hRluc vector used to correct for transfection efficiency was a product of Promega (Mannheim, Germany).

For the mammalian two-hybrid assays we cloned plasmids coding for fusion proteins of the Gal4 DNA-binding domain and either mouse NCoR amino acids 1942-2453 (Gal4-NCoRc) or mouse NCoR amino acids 1-695 (Gal4-NCoRn) according to the manufacturer's instructions using the Flexi Vector Systems (Promega). Plasmids for fusion proteins of the VP16 activation domain and the AR amino acids 1-640 (VP16ARdel) or full length AR (VP16-AR) were similarly cloned, as well as the VP16-tagged NCoR C-terminus (VP16-NCoRc). For Gal4 fusion expression plasmids, we used the vector pFN11A (Gal4) from Promega. pFN10A (VP16, Promega) was used for the VP16 fusion expression plasmids. NCoR fusion plasmids were cloned using pCMX-NCoR as a template and pSG5-AR served as template for AR fusions. pGL4.31 (Promega) was used as luciferase reporter in the two-hybrid assays.

Cell culture. The cell lines, PC-3, LNCaP, COS-1 were obtained from the ATCC (Manassas, VA, USA). DU-CaP and V-CaP were kindly provided by Professor Culig (Innsbruck Medical University). PC-3, LNCaP and COS-1 were grown in RPMI1640 (PAA Laboratories GmbH, Pasching, Austria) in an atmosphere with $5 \% \mathrm{CO}_{2}$. DU-CaP and $\mathrm{V}-\mathrm{CaP}$ cells were cultured in DMEM containing $1 \%$ Glutamax $\left(\right.$ Gibco $^{\circledR}$, distributed by Invitrogen, Darmstadt, Germany). Both media were supplemented with $10 \%$ fetal bovine serum (FBS) and $1 \%$ penicillin/streptomycin (PAA Laboratories $\mathrm{GmbH}$ ).
Dihydrotestosterone (DHT) (Sigma-Aldrich, Taufkirchen, Germany), was used as a $1 \mathrm{mM}$ stock solution in ethanol. During experiments, cells were maintained in RPMI-1640 with $2.5 \%$ steroid-free dextran charcoal treated serum (v/v) (FBSdcc Biowest, Nuaillé, France) and antibiotics in the presence or absence of DHT. Plasticware was purchased from Sarstedt (Nürmbrecht, Germany).

Transfection and dual luciferase reporter assay. For transient transfection cells were grown in 24-well plates. COS-1, PC-3, DU-CaP and V-CaP cells were transfected using the PolyFect transfection reagent (Qiagen, Hilden, Germany) according to the manufacturer's instructions. Attractene transfection reagent (Qiagen) was used for transfection of LNCaP cells.

COS-1 and PC-3 cells were co-transfected with pSG5-AR (62.5 ng/well) and with either PSA61-luc (200 ng/well) or ARE-luc (200 ng/well). AR-positive LNCaP cells were routinely transfected with the pGL3E-Probasin reporter (200 ng/well) as previously described (22). pGL4hRluc used to correct for transfection efficiency was co-transfected in every experiment (80 ng/well). After transfection, cells were grown in RPMI1640 with 5\% FBSdcc and treated with $10 \mathrm{nM}$ DHT. Reporter activity was analyzed after $24 \mathrm{~h}$ using the Dual Luciferase Reporter Assay system (Promega). Transfection of increasing amounts of NCoR expression plasmid was balanced by the corresponding empty vector. Transfection experiments were repeated at least three times and performed in triplicate, unless otherwise stated.

Mammalian two hybrid assay. To investigate NCoR/AR interaction we used the CheckMate/FlexiVector Mammalian Two-Hybrid system from Promega. Cells were grown in 24-well plates and co-transfected with pAct-AR, pGL4.31 and either Gal4-NCoRc, Gal4-NCoRn or pCMX-NCoR (each $200 \mathrm{ng} /$ well). After transfection, cells were cultured and luciferase activities were determined as described above.

Western blot analysis. Proteins were extracted from cells using RIPA buffer as previously described (23). Proteins (25 $\mu \mathrm{g}$ lysate) were separated by SDS polyacrylamide gel electrophoresis. For Western blot analysis proteins were transferred to a nitrocellulose membrane (BioTrace NT; Pall Life Sciences, Dreieich, Germany) by tank blotting in transfer buffer [20 mM Tris-HCl, pH 8.7, $150 \mathrm{mM}$ glycine and 20\% (v/v) methanol]. Membranes were blocked in phosphate-buffered saline (PBS) with $0.1 \%$ Tween-20 (PBS-T) and 5\% BSA for $1 \mathrm{~h}$ at room temperature. The membrane was incubated with the primary antibody in PBS-T with $1 \%$ BSA overnight at $4{ }^{\circ} \mathrm{C}$. $\beta$-actin was detected with the mouse monoclonal antibody mAbcam 8224 (Abcam, Cambridge, UK) at a dilution of 1:20,000. AR was detected with the mouse monoclonal antibody AR441 (Dako Deutschland GmbH, Hamburg, Germany) at a dilution of 1:1,000. The membrane was washed with PBS-T three times before incubating with the peroxidase-coupled secondary antibody at a dilution of 1:2,000 in PBS-T with $1 \%$ BSA. Signals were visualized by the SuperSignal West Pico Chemiluminescent Substrate from Pierce (Rockford, USA).

Statistical analysis. All experiments were carried out at least three times. Data are reported as mean \pm SD. Analysis was 

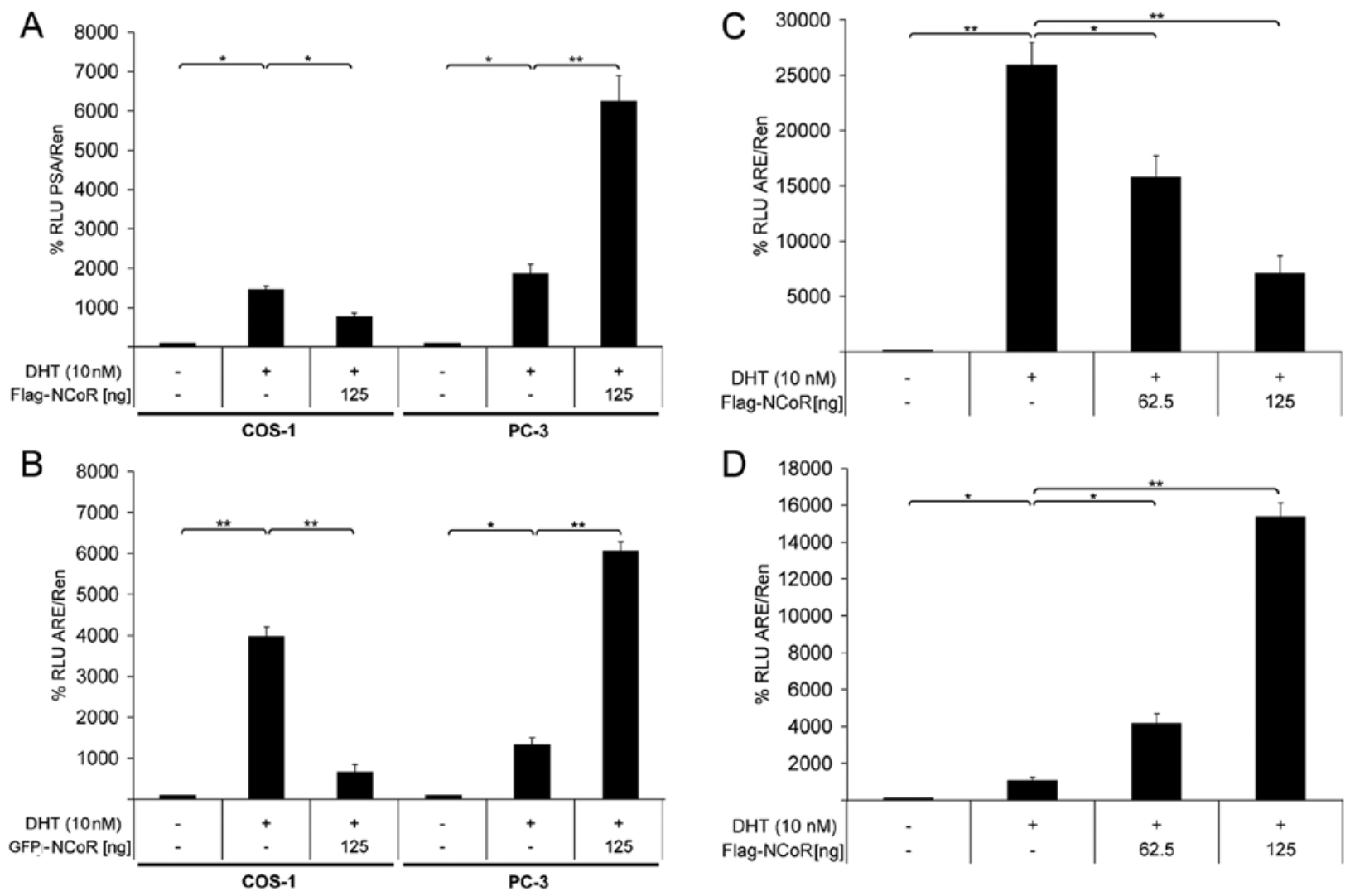

Figure 1. NCoR fails to repress AR transcriptional activity in PC-3 cells. COS-1 and PC-3 cells were co-transfected with pSG5-AR, a Renilla luciferase construct to correct for transfection efficiency, a luciferase reporter gene construct and an NCoR expression vector. Cells were treated with DHT and $24 \mathrm{~h}$ later luciferase activities were determined using the dual luciferase assay system (Promega, Mannheim, Germany). (A) The transfected luciferase reporter gene was controlled by the androgen responsive PSA-promoter. The NCoR expression vector was pCMX-Flag-NCoR. One representative result out of 3 independent experiments is shown. (B) Cells were co-transfected with GFP-NCoR and with a luciferase reporter gene controlled by an ARE. This confirmatory experiment was repeated twice in triplicates and one representative result is shown. (C) COS-1 and (D) PC-3 cells were co-transfected as in (B) but with increasing amounts of pCMX-Flag-NCoR. One representative result of at least 4 independent experiments is shown. Error bar $=\mathrm{SD} . \mathrm{n}=3$. ${ }^{*} \mathrm{P}<0.05 ;{ }^{* * *} \mathrm{P}<0.01$.

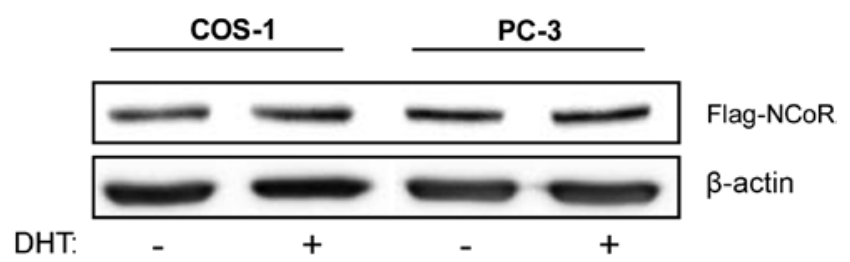

Figure 2. Expression of Flag-NCoR. Expression of the NCoR-Flag fusion protein was controlled by Western blot analysis. Cells were transiently transfected with pCMX-Flag-NCoR, treated with $10 \mathrm{nM}$ DHT (+) or vehicle (-) and harvested $48 \mathrm{~h}$ after transfection. Whole cell lysates $(25 \mu \mathrm{g})$ were separated by electrophoresis on a $6 \%$ SDS-polyacrylamide gel and transferred onto a nitrocellulose membrane. Western blot analysis was performed as described The NCoR-Flag fusion protein was detected with anti-Flag M2 monoclonal antibody (Sigma, Hamburg, Germany).

performed with Student's t-test with $\mathrm{P}<0.05$ considered as significant.

\section{Results}

$N C o R$ and $S M R T \alpha / \tau$ repress $A R$ transcriptional activity in COS-1 but not in PC-3 cells. AR-negative COS-1 and PC-3 cells were transiently transfected with expression plasmids coding for wild-type AR, Flag-tagged NCoR and a luciferase reporter gene plasmid containing an androgen responsive promoter. Additionally a Renilla luciferase expression plasmid was co-transfected for normalization. Subsequently, cells were treated with $10 \mathrm{nM}$ DHT for $24 \mathrm{~h}$. Transactivation was measured using a dual luciferase reporter system. Treating COS-1 as well as PC-3 cells with an androgen (DHT) resulted in a 15-fold (COS-1) or 18-fold (PC-3) increase in PSA promoter activity. Interestingly, co-transfection of the co-repressor NCoR reduced AR transcriptional activity in COS- 1 cells by $50 \%$, whereas in PC-3 cells NCoR co-transfection led to an increase of PSA promoter activity (Fig. 1A). Similar results were obtained by using an ARE-luc reporter gene construct in combination with a GFP-NCoR expression plasmid (Fig. 1B). In order to test whether the discrepancy between COS-1 and PC-3 cells is not due to differential expression or localization of NCoR we analyzed NCoR protein expression by Western blot analysis and immunofluorescence staining detecting the Flag epitope of exogenous Flag-NCoR. In both cell lines the NCoR-Flag fusion protein was expressed in similar amounts (Fig. 2) and was localized in the nucleus (data not shown). Transfection of increasing amounts of Flag-NCoR expression plasmid led to a dose-dependent decrease in AR transcriptional activity in COS-1 cells (Fig. 1C), whereas in PC-3 cells reporter gene activity was up-regulated (Fig. 1D). Transfection of up to $500 \mathrm{ng}$ NCoR expression plasmid had a similar effect as 

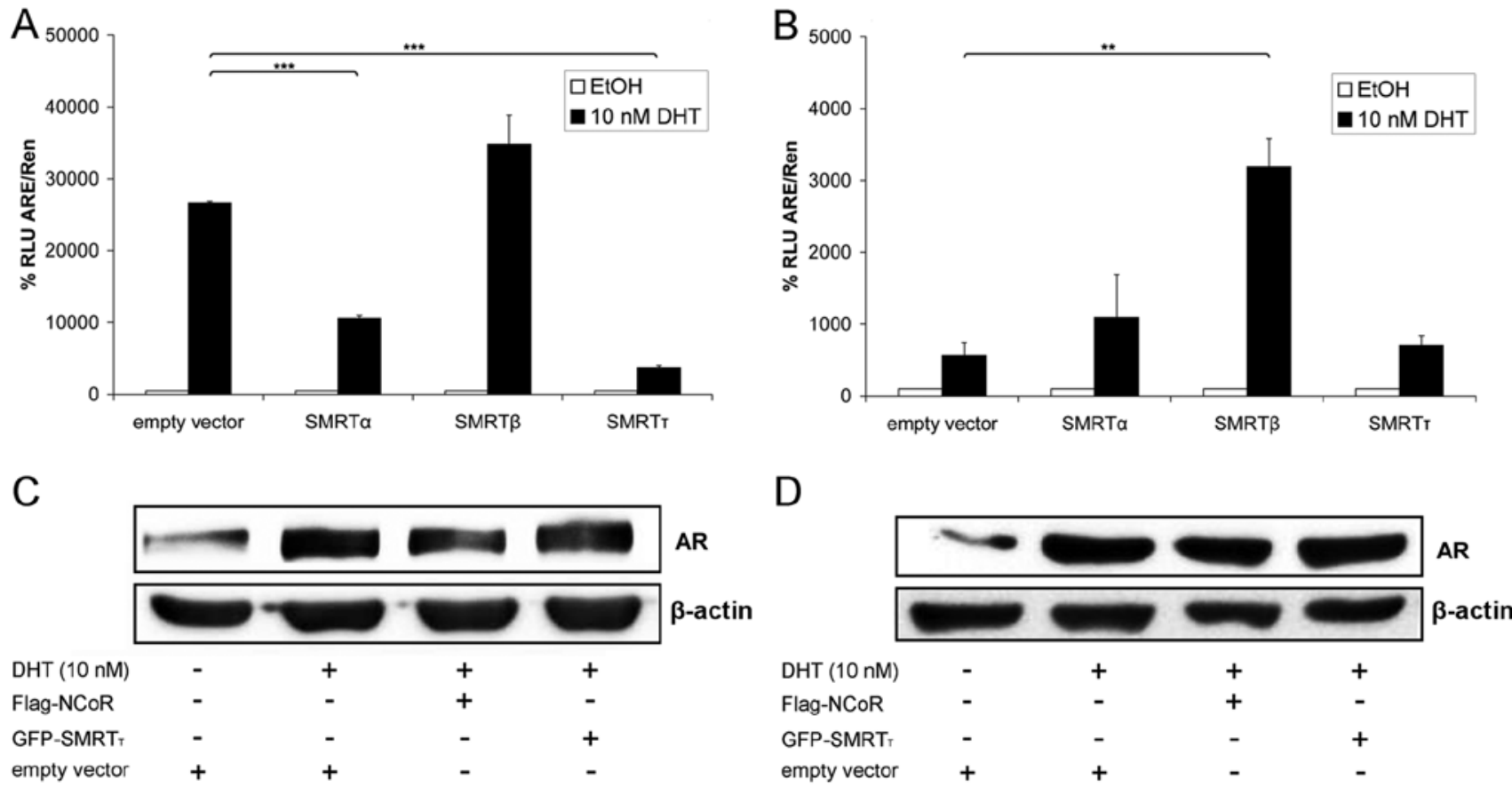

Figure 3. SMRT $\alpha$ and $\tau$ repress AR transcriptional activity in COS-1, but not in PC-3 cells. (A) COS-1 and (B) PC-3 cells were co-transfected with pSG5-AR, a Renilla luciferase construct to correct for transfection efficiency, a luciferase reporter gene controlled by an ARE and with expression constructs for either SMRT $\alpha$, SMRT $\beta$ or SMRT $\tau$. Cells were treated with DHT and after $24 \mathrm{~h}$ luciferase activities were determined. One representative result of at least 3 independent experiments is shown. (C) COS-1 and PC-3 (D) cells were seeded into 6-well plates. Cells were co-transfected with 500 ng/well pSG5-AR and $1 \mu \mathrm{g} /$ well of either pCMX-Flag-NCoR, pCMV-GFP-SMRT $\tau$ expression vector or an empty vector. After transfection, cells were treated with DHT for $24 \mathrm{~h}$ and harvested. Whole cell lysates $(25 \mu \mathrm{g})$ were separated by electrophoresis on a $10 \%$ SDS-polyacrylamide gel and transferred onto a nitrocellulose membrane. Western blot analysis was performed as described. Error bar $=$ SD. $n=3$. ${ }^{* * *} \mathrm{P}<0.01 ;{ }^{* * * *} \mathrm{P}<0.001$.
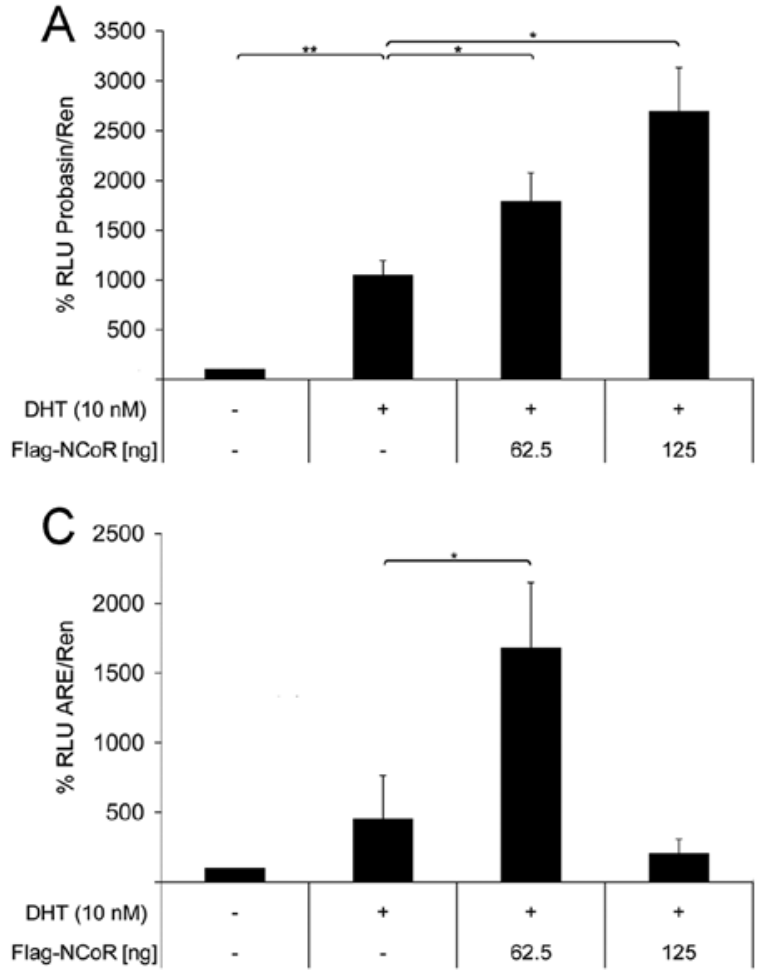

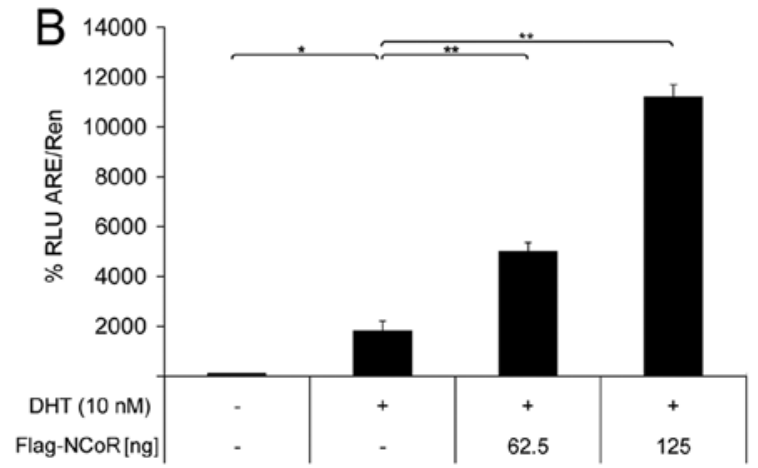

D
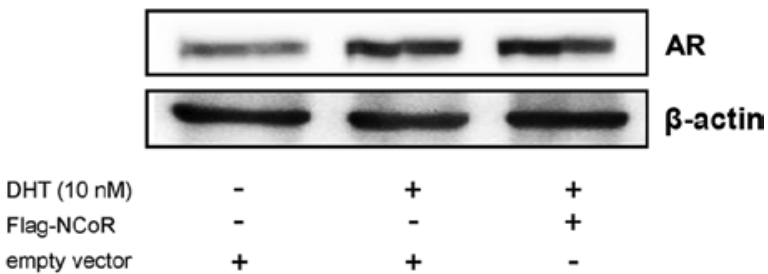

Figure 4. NCoR does not repress endogenous AR transcriptional activity. (A) LNCaP, (B) DU-CaP or (C) V-CaP cells were transfected with different amounts of NCoR expression vector. A Renilla luciferase construct to correct for transfection efficiency and a luciferase reporter gene controlled by an androgen responsive promoter (probasin or ARE) were co-transfected. Cells were treated with DHT and luciferase activities were determined. For LNCaP cells one representative of at least 3 independent experiments is shown. The confirmatory experiments with DU-CaP and V-CaP cells were repeated twice for each cell line. (D) LNCaP cells were seeded into 6-well plates and transfected with $1 \mu \mathrm{g} /$ well pCMX-Flag-NCoR expression vector or an empty vector. After transfection cells were treated with DHT for $24 \mathrm{~h}$ and harvested. Whole cell lysates $(25 \mu \mathrm{g})$ were separated by electrophoresis on a $10 \%$ SDS-polyacrylamide gel and transferred onto a nitrocellulose membrane. Western blot analysis was performed as described. Error bar $=\mathrm{SD} . \mathrm{n}=3 .{ }^{*} \mathrm{P}<0.05$; ${ }^{* *} \mathrm{P}<0.01$. 


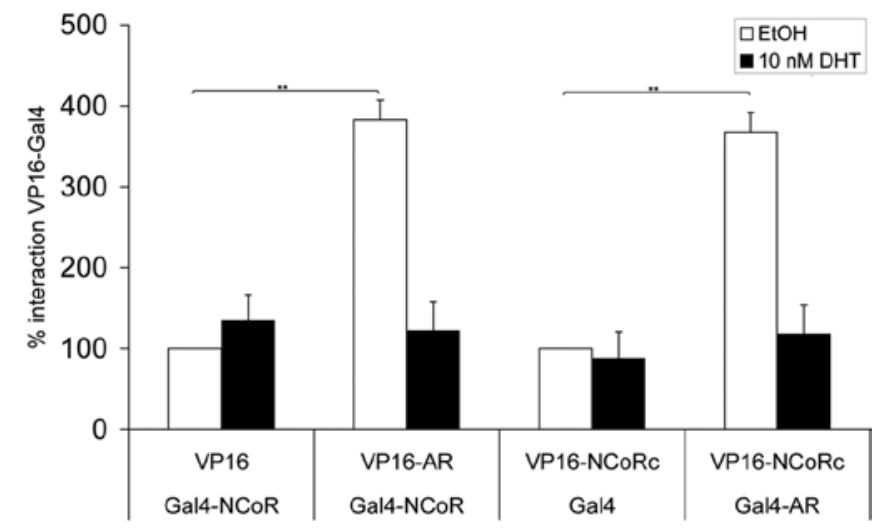

Figure 5. M2H control experiment: NCoR full length protein as well as NCoR C-terminus interacts with AR in COS-1 cells. COS-1 cells were co-transfected with the indicated plasmids as described in Materials and methods. pGL4.31 was additionally transfected and used as a reporter gene. Cells were treated with DHT and luciferase activities were determined after 24 h. Error bar $=$ SD. $n=3 .{ }^{* *} \mathrm{P}<0.01$.

transfection of $125 \mathrm{ng}$ NCoR in both COS-1 and PC-3 cells (data not shown). The activity of the Renilla luciferase used for normalization remained unchanged upon co-repressor transfection in both cell lines (not shown). Based on these results we investigated whether the inability of NCoR to repress androgen-responsive promoters in PC-3 cells is specific to this repressor. The NCoR related co-repressor SMRT has also been described to repress AR transcriptional activity (16). We therefore conducted similar transfection experiments with SMRT. Interestingly, the SMRT $\alpha$ as well as the SMRT $\tau$ isoforms failed to repress AR transcriptional activity on the PSA promoter in PC-3 (Fig. 3B) but not in COS-1 cells (Fig. 3A). In contrast, the SMRT $\beta$ isoform activated $\mathrm{AR}$ transcriptional activity in both cell lines (Fig 3A and B). Co-transfection of SMRT $\alpha$ or SMRT $\tau$ in PC-3 cells did not result in a repression of AR transactivation. As the up-regulation of AR transcriptional activity could be due to an increase of AR protein levels upon transfection of co-repressors we verified the amount of AR protein by Western blotting. Fig. 3C and D show an increase in AR protein after DHT treatment in each of the cell lines. In the presence of the hormone, AR levels remained unchanged after transfection of NCoR or SMRT $\tau$ expression vectors compared to the empty vector control. Therefore, the increase in AR transactivation activity after transfection of co-repressors is not due to a rise in AR protein level.

$N C o R$ does not repress endogenous AR transcriptional activity. To test whether the failure of NCoR and SMRT to repress AR transcriptional activity is a PC-3-specific phenomenon which is due to the transient expression of the AR, we subsequently transfected the AR-positive LNCaP prostate cancer cell line. LNCaP cells were co-transfected with an androgen-responsive probasin promoter-driven luciferase reporter gene to determine transcriptional activity of the endogenous AR in the presence of increasing amounts of the NCoR expression construct. As expected, DHT treatment induced AR transcriptional activity in $\mathrm{LNCaP}$ cells. Co-transfection of NCoR in LNCaP cells enhanced the transcriptional activity of the endogenous AR
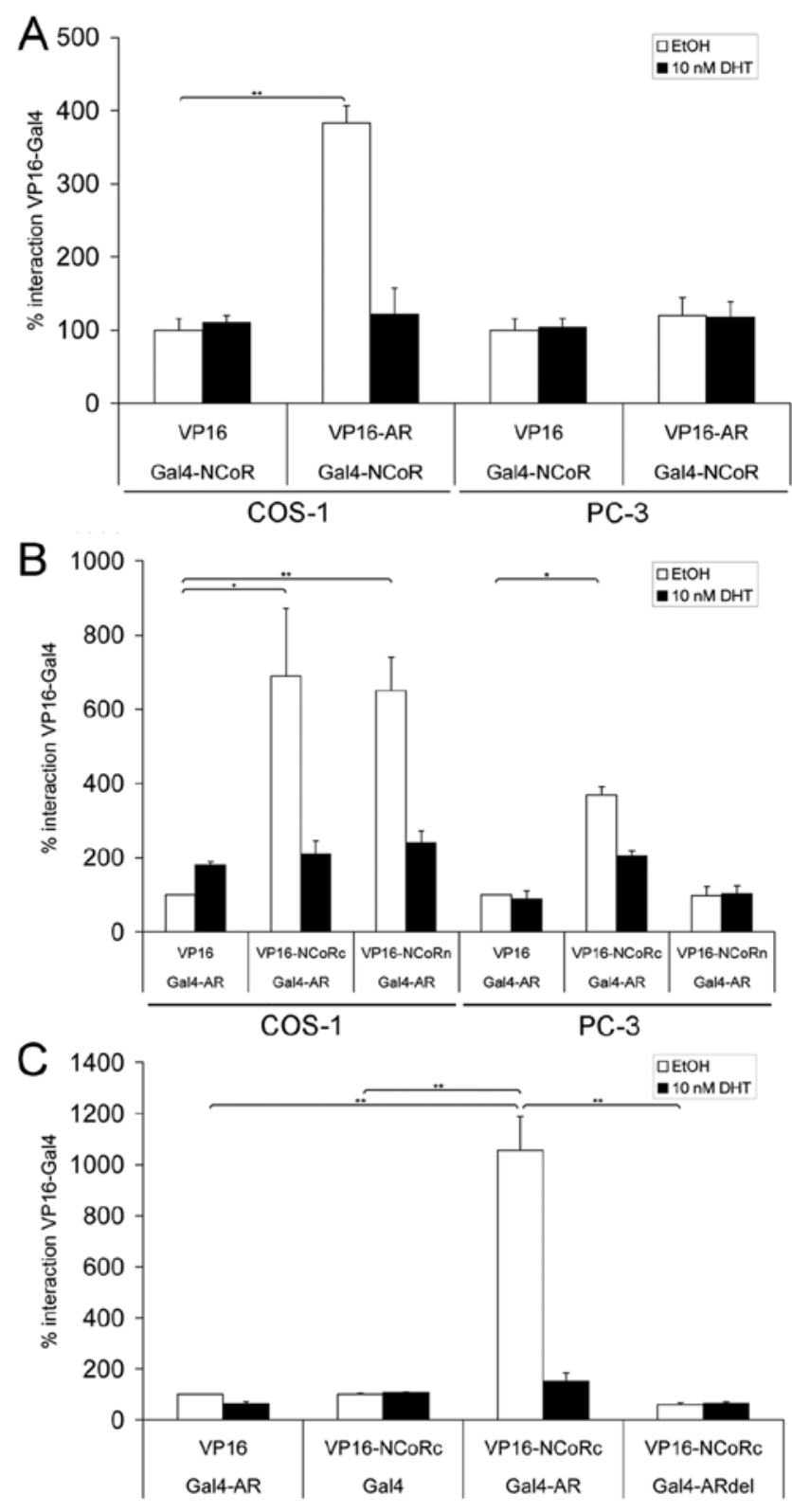

Figure 6. Impaired AR-NCoR interaction in PC-3 cells. COS-1 and PC-3 cells were co-transfected with expression plasmids for (A) Gal4-NCoR full length protein and VP16-AR as well as plasmids encoding (B) VP16-NCoR C- (VP16-NCoRc) or N-terminus (VP16-NCoRn) and Gal4-AR or the corresponding empty vectors. pGL4.31 was additionally transfected and used as a reporter gene. Cells were treated with DHT and after $24 \mathrm{~h}$ luciferase activities were determined. (C) COS-1 cells were co-transfected with expression plasmids for VP16-NCoR C-terminus and a truncated AR (Gal4ARdel). Error bar $=$ SD. $n=3 .{ }^{*} \mathrm{P}<0.05 ;{ }^{* *} \mathrm{P}<0.01$.

up to 2.7-fold depending on the transfected amount (Fig. 4A). The endogenous AR protein levels in the LNCaP cells (Fig. 4D) were not increased after NCoR transfection. Similarly, NCoR was not able to repress AR activity in PC-3 cells when the probasin promoter was transfected (data not shown). To clarify whether this effect is due to the mutation of the AR which is endogenously expressed in $\mathrm{LNCaP}$ cells, the repression by $\mathrm{NCoR}$ was examined in additional PCa cell lines DU-CaP and $\mathrm{V}-\mathrm{CaP}$ expressing wild-type AR. NCoR was not able to repress endogenous AR activity in these cell lines (Fig. 4B and C). In summary, $\mathrm{NCoR}$ failed to repress transactivation of 
the AR in the AR-positive LNCaP, DU-CaP and V-CaP cells as well as in PC-3 cells with an AR-expression construct.

Impaired AR-NCoR interaction in PC-3 cells. In order to determine whether the inability of NCoR to repress AR transcriptional activity is due to impaired repressor-receptor interaction we performed a mammalian two hybrid (M2H) assay. First we performed the interaction assay in COS-1 cells (Fig 5). The NCoR full length protein as well as the NCoR $\mathrm{C}$-terminus interacted with the AR in the absence of hormone. The interaction was decreased by $75 \%$ by the addition of DHT. Since the AR has been described to interact via its C-terminus with the co-repressor (18) we constructed a C-terminal deleted AR to verify our M2H data. The shortened AR was unable to interact with the co-repressor (Fig. 6C). The interaction of $\mathrm{NCoR}$ with the AR was compared in COS-1 and PC-3 cells using a full-length $\mathrm{NCoR}$ expression construct and expression constructs encoding the NCoR N- or C-terminus. Full length $\mathrm{NCoR}$ interacted with the AR in COS-1 cells but not in PC-3 cells (Fig. 6A). Additionally to the NCoR C-terminal nuclear receptor interaction domains, an $\mathrm{N}$-terminal interaction domain has recently been described (27). Therefore we studied the interaction of the $\mathrm{NCoR} \mathrm{N-}$ and C-terminus with AR. Interestingly the NCoR C-terminus interacted with the receptor in COS-1 as well as in PC-3 cells, whereas an interaction with the NCoR N-terminus only occurred in COS-1 cells (Fig. 6B).

\section{Discussion}

In the present study we analyzed the effects of a transient overexpression of the co-repressors NCoR and SMRT on AR signaling in COS- 1 and PCa cells. As expected, NCoR and $\mathrm{SMRT} \alpha / \tau$ inhibited AR transactivation in COS- 1 cells. However, in contrast to these findings, AR transactivation was increased in PCa cell lines after overexpression of both co-repressors. Although SMRT and NCoR are well studied co-repressors, the majority of reporter gene assays investigating their influence on AR transcriptional activity has been performed in non-prostate cell lines. In human embryonic kidney 293 cells SMRT inhibits DHT-dependent AR transcriptional activation. Similarly, in CV1 cells NCoR represses DHT-dependent transcriptional activation and interacts directly with the AR (18). In a further study, NCoR was shown to repress AR transcriptional activity in COS-1 but not in HeLa cells (17). This observation is consistent with our findings showing repression of AR transcriptional activity in COS-1 cells but not in PCa cells. To our knowledge this is the first report showing an enhancement, rather than a repression of AR signaling in prostate cancer cell lines following $\mathrm{NCoR} / \mathrm{SMRT} \alpha / \tau$ overexpression. The differences in AR transactivation in COS-1 and PCa cells following SMRT/NCoR overexpression probably reflect tissue-specific properties of the different cell types. Indeed, cell-specific differences in steroid receptor transactivation following SMRT $\tau$ overexpression have been also reported for estrogen receptor $\alpha(E R \alpha)$ (24). Transient transfection of SMRT $\tau$ in HeLa and MCF-7 cells increased ER $\alpha$ transcriptional activity, whereas in HepG2 cells overexpression of SMRT $\mathrm{Tad}$ no effect. The co-activation of ER $\alpha$ by SMRT in MCF-7 cells was due to the formation of a ternary complex between the co-activator SRC-3, SMRT and ER $\alpha$. The direct interaction of SRC-3 and SMRT is thought to promote hormonedependent binding of SRC-3 to ER $\alpha$ (25). Interestingly, NCoR was also shown to interact with SRC-3 (26). Thus it may be conceivable that a similar scenario is responsible for the enhancement of AR transcriptional activity after transient overexpression of $\mathrm{NCoR}$ in $\mathrm{PC}-3$ cells. Indeed the $\mathrm{NCoR}$ C-terminus was recently shown to be involved in SRC-3 binding (26) suggesting that NCoR might be responsible for the recruitment of SRC-3 to the AR.

In a recent paper Varlakhanova et al (26) reported a new receptor interaction motif in the amino terminal region of the SMRT $\alpha$ isoform. This motif also occurred in the related co-repressor NCoR and interacted with ER $\alpha$ (27). To our knowledge this is the first report showing an interaction of the $\mathrm{NCoR} \mathrm{N}$-terminus with the AR. Whereas the $\mathrm{NCoR} \mathrm{C}$-terminus interacted with the AR in both COS-1 and PC-3 cells, its $\mathrm{N}$-terminal part was only able to bind in COS-1 and not in PC-3 cells. The diminished binding of the NCoR N-terminus to the AR in PC-3 cells may prevent repression of the AR transcriptional activity, but still allows the binding of SRC-3 thereby leading to AR co-activation. We hypothesize that both the $\mathrm{N}$ - and the C-terminal receptor interaction domains are necessary for NCoR mediated AR repression.

The importance of the co-repressor $\mathrm{N}$-terminus is highlighted by recent experiments using the SMRT $\beta$ isoform. This SMRT-isoform lacks N-terminal amino acids harboring one of the classical known repression domains and therefore shows an impaired repression activity $(15,26,27)$. Moreover, SMRT $\beta$ is lacking the newly described N-terminal receptor interaction domain (26). Accordingly, overexpression of the SMRT $\beta$ isoform was demonstrated to increase AR transcriptional activity in COS-1 and in PC-3 cells. This is consistent with the results from Cote $e t$ al showing an increase of wildtype RAR $\alpha$ in Jurkat cells upon SMRT $\beta$ overexpression (28). The fact that we are not able to show an interaction of the NCoR full length protein in PC-3 cells although the NCoR C-terminus binds to the AR may be due to limitations of the mammalian two-hybrid assay. The proximity between the VP16-AD and Gal4-DBD fusions is probably not sufficient enough to activate the reporter gene because of the size of their fusion partners, NCoR and AR. However, in COS-1 cells the additional interaction via the NCoR N-terminus brings the VP16 and the Gal4 closer together resulting in reporter gene activation.

In PCa cell lines as well as in primary cultures of advanced prostate cancer, the corepressors SMRT, NCoR and Prohibitin were reported to be overexpressed (11-14). At first sight an up-regulation of the co-repressors seems unlikely to contribute to tumor progression. However, our results support the assumption that NCoR/SMRT repression activity depends on proper interaction of their $\mathrm{N}$ - and $\mathrm{C}$-terminal interaction domains, which may be influenced by the cell type. As shown in this study, NCoR and SMRT are likely to increase AR transcriptional activity in PCa cells. Therefore, up-regulation of co-repressors in prostate cancer may result in increased AR activity and in consequence provides a proliferation advantage for prostate cancer cells. In summary our in vitro findings show that the effects of NCoR and SMRT on AR signaling vary according to a the cell type and highlight the complexity of AR-co-repressor interaction in human PCa cells. 


\section{Acknowledgements}

We thank PD Dr M. Cronauer for helpful suggestions and Mrs. S. Schmidt for skillful technical assistance.

\section{References}

1. Rinnab L, Hessenauer A, Schutz SV, Schmid E, Kufer R, Finter F Hautmann RE, Spindler KD and Cronauer MV: Role of androgen receptors in hormone-refractory prostate cancer: molecular basics and experimental therapy approaches. Urologe A 47: 314-325, 2008 (In German).

2. Hobisch A, Culig Z, Radmayr C, Bartsch G, Klocker H and Hittmair A: Distant metastases from prostatic carcinoma express androgen receptor protein. Cancer Res 55: 3068-3072, 1995.

3. Feldman BJ and Feldman D: The development of androgenindependent prostate cancer. Nat Rev Cancer 1: 34-45, 2001.

4. Gregory CW, He B, Johnson RT, Ford OH, Mohler JL, French FS and Wilson EM: A mechanism for androgen receptor-mediated prostate cancer recurrence after androgen deprivation therapy. Cancer Res 61: 4315-4319, 2001

5. Culig Z, Comuzzi B, Steiner H, Bartsch G and Hobisch A: Expression and function of androgen receptor coactivators in prostate cancer. J Steroid Biochem Mol Biol 92: 265-271, 2004.

6. Chmelar R, Buchanan G, Need EF, Tilley W and Greenberg NM Androgen receptor coregulators and their involvement in the development and progression of prostate cancer. Int J Cancer 120: 719-733, 2007.

7. Wang C, Hsu L and Chang C: Androgen receptor corepressors: an overview. Prostate 63: 117-130, 2005.

8. Belandia B, Powell SM, Garcia-Pedrero JM, Walker MM, Bevan CL and Parker MG: Hey1, a mediator of notch signaling, is an androgen receptor corepressor. Mol Cell Biol 25: 1425-1436, 2005.

9. Gamble SC, Chotai D, Odontiadis M, Dart DA, Brooke GN, Powell SM, Reebye V, Varela-Carver A, Kawano Y, Waxman J and Bevan CL: Prohibitin, a protein downregulated by androgens, represses androgen receptor activity. Oncogene 26: 1757-1768, 2007.

10. Gamble SC, Odontiadis M, Waxman J, Westbrook JA, Dunn MJ, Wait R, Lam EW and Bevan CL: Androgens target prohibitin to regulate proliferation of prostate cancer cells. Oncogene 23 : 2996-3004, 2004

11. Ummanni R, Junker H, Zimmermann U, Venz S, Teller S, Giebel J, Scharf C, Woenckhaus C, Dombrowski F and Walther R: Prohibitin identified by proteomic analysis of prostate biopsies distinguishes hyperplasia and cancer. Cancer Lett 266: 171-185, 2008.

12. Khanim FL, Gommersall LM, Wood VH, Smith KL, Montalvo L, O'Neill LP, Xu Y, Peehl DM, Stewart PM, Turner BM and Campbell MJ: Altered SMRT levels disrupt vitamin D3 receptor signalling in prostate cancer cells. Oncogene 23: 6712-6725, 2004.

13. Ting HJ, Bao BY, Reeder JE, Messing EM and Lee YF: Increased expression of corepressors in aggressive androgenindependent prostate cancer cells results in loss of 1alpha,25-dihydroxyvitamin D3 responsiveness. Mol Cancer Res 5: 967-980, 2007.

14. Li J, Wang J, Wang J, Nawaz Z, Liu JM, Qin J and Wong J: Both corepressor proteins SMRT and N-CoR exist in large protein complexes containing HDAC3. EMBO J 19: 4342-4350, 2000.
15. Goodson M, Jonas BA and Privalsky MA: Corepressors: custom tailoring and alterations while you wait. Nucl Recept Signal 3: $\mathrm{e} 003,2005$.

16. Liao G, Chen LY, Zhang A, Godavarthy A, Xia F, Ghosh JC, $\mathrm{Li} \mathrm{H}$ and Chen JD: Regulation of androgen receptor activity by the nuclear receptor corepressor SMRT. J Biol Chem 278: 5052-5061, 2003.

17. Agoulnik IU, Krause WC, Bingman WE, Rahman HT, Amrikachi M, Ayala GE and Weigel NL: Repressors of androgen and progesterone receptor action. J Biol Chem 278: 31136-31148, 2003.

18. Cheng S, Brzostek S, Lee SR, Hollenberg AN and Balk SP: Inhibition of the dihydrotestosterone-activated androgen receptor by nuclear receptor corepressor. Mol Endocrinol 16: 1492-1501, 2002.

19. Jonas BA and Privalsky ML: SMRT and N-CoR corepressors are regulated by distinct kinase signaling pathways. J Biol Chem 279: 54676-54686, 2004.

20. Tiefenbach J, Novac N, Ducasse M, Eck M, Melchior F and Heinzel T: SUMOylation of the corepressor N-CoR modulates its capacity to repress transcription. Mol Biol Cell 17: 1643-1651, 2006.

21. Peters I, Weidemann W, Romalo G, Knorr D, Schweikert HU and Spindler KD: An androgen receptor mutation in the direct vicinity of the proposed C-terminal alpha-helix of the ligand binding domain containing the AF-2 transcriptional activating function core is associated with complete androgen insensitivity. Mol Cell Endocrinol 148: 47-53, 1999.

22. Cronauer MV, Ince Y, Engers R, Rinnab L, Weidemann W, Suschek CV, Burchardt M, Kleinert H, Wiedenmann J, Sies H, Ackermann R and Kroncke KD: Nitric oxide-mediated inhibition of androgen receptor activity: possible implications for prostate cancer progression. Oncogene 26: 1875-1884, 2007.

23. Schneider CC, Hessenauer A, Montenarh M and Gotz C: p53 is dispensable for the induction of apoptosis after inhibition of protein kinase CK2. Prostate 70: 126-134, 2010.

23. Peterson TJ, Karmakar S, Pace MC, Gao T and Smith CL: The silencing mediator of retinoic acid and thyroid hormone receptor (SMRT) corepressor is required for full estrogen receptor alpha transcriptional activity. Mol Cell Biol 27: 5933-5948, 2007.

24. Karmakar S, Gao T, Pace MC, Oesterreich S and Smith CL: Cooperative activation of cyclin D1 and progesterone receptor gene expression by the SRC-3 coactivator and SMRT corepressor. Mol Endocrinol 24: 1187-1202, 2010.

25. Li X, Kimbrel EA, Kenan DJ and McDonnell DP: Direct interactions between corepressors and coactivators permit the integration of nuclear receptor-mediated repression and activation. Mol Endocrinol 16: 1482-1491, 2002.

26. Varlakhanova N, Snyder C, Jose S, Hahm JB and Privalsky ML: Estrogen receptors recruit SMRT and $\mathrm{N}-\mathrm{CoR}$ corepressors through newly recognized contacts between the corepressor $\mathrm{N}$ terminus and the receptor DNA binding domain. Mol Cell Biol 30: 1434-1445, 2010.

27. Ordentlich P, Downes M, Xie W, Genin A, Spinner NB and Evans RM: Unique forms of human and mouse nuclear receptor corepressor SMRT. Proc Natl Acad Sci USA 96: 2639-2644, 1999.

28. Cote S, McNamara S, Brambilla D, Bianchini A, Rizzo G, del Rincon SV, Grignani F, Nervi C and Miller Jr WH: Expression of SMRTbeta promotes ligand-induced activation of mutated and wild-type retinoid receptors. Blood 104: 4226-4235, 2004. 